Research Article

\title{
Application of Blockchain for Internet of Things: A Bibliometric Analysis
}

\author{
Ruijun Duan $(1)$ and Li Guo \\ School of Economics and Commerce, Henan University of Technology, Zhengzhou 450001, China \\ Correspondence should be addressed to Ruijun Duan; duanrj52058@126.com
}

Received 18 January 2021; Revised 28 March 2021; Accepted 23 April 2021; Published 29 April 2021

Academic Editor: Chien Ming Chen

Copyright (c) 2021 Ruijun Duan and Li Guo. This is an open access article distributed under the Creative Commons Attribution License, which permits unrestricted use, distribution, and reproduction in any medium, provided the original work is properly cited.

As a disruptive emerging technology, the Internet of things (IoT) has rapidly developed, but its privacy risks and security vulnerabilities are still key challenges. The decentralized and distributed architecture of blockchain has the potential to satisfy IoT privacy and security requirements. This gives birth to the new domain of blockchain for IoT (BIoT). BIoT will cause significant transformations across several industries, paving the way for new business models. Based on the Science Citation Index Expanded (SCIE) and Social Sciences Citation Index (SSCI) databases in Web of Science (WoS) Core Collection, this study aims to explore the research trends and cooperation in the field of BIoT using the bibliometric method. The results indicate that the publications in this field have increased significantly from 2016 to 2020, with China and the USA being the most productive and influential countries. Keyword co-occurrence analysis shows that the most important research topics are as follows: security issues, core technologies, application dimensions, and transaction processes. Text mining analysis indicates that future research directions for BloT will focus more on both computing paradigms and key applications. This study will provide researchers with a greater understanding on the state of the art of BIoT and will serve as a reference for researchers engaging in this field to identify their own future research directions.

\section{Introduction}

Internet of things (IoT) applications have been rapidly increasing in recent years and are currently playing a key role in the development of society. In fact, through the connection of vehicles, household appliances, and other electronic devices to the Internet, IoT will create more comfortable living conditions for humans through many applications coming from various industrial domains such as smart home, smart healthcare, transportation, agriculture, retail, and wearable [1]. Cisco predicts that the share of machine-to-machine (M2M) connections will increase from $33 \%$ in 2018 to $50 \%$ in 2023 , i.e., 14.7 billion connections, with connected home applications accounting for nearly half this share, while connected car applications will grow the fastest at a CAGR of 30\% [2]. Currently, most IoT solutions rely on a centralized server-client paradigm, which involves connecting to cloud servers through the Internet [3].
However, centralized architectures are susceptible to the malicious manipulation of information by untrusted entities, which may in turn result in the exchange of tampered and forged information [4]. On the other hand, cloud services that store, forward, and process IoT data are vulnerable to network attacks such as false data injection, data tampering, and single node failures [5]. To sum up, the major concerns regarding the development of IoT are security and privacy. In [6], it is pointed out that blockchainbased decentralized architectures provide solutions to the problem of IoT application security.

Blockchain was originally invented as the underlying technology of bitcoin, but its potential applications have gone far beyond the scope of cryptocurrencies. The emergence of blockchain marks a breakthrough in decentralized technologies. Since trade on the Internet relies almost entirely on third-party financial institutions to handle electronic payments, the existence of financial intermediaries 
increases transaction costs, limits the minimum actual transaction size, and there exist many potential malicious activity possibilities, such as fraud [7]. As a distributed technology, blockchain is created to overcome the inherent weakness of this trust-based model, because it is an electronic payment system based on proof of encryption rather than trust. Blockchain allows peer-to-peer transactions through decentralized networks using data encryption, time stamps, tree structures, and consensus mechanisms without having to rely on third-parties [8]. It also provides public and full access to the history of transactions, allows access to all transactions that have occurred since the first transaction, and their occurrence can be verified by any entity at any time $[3,9]$. Blockchain technology is conducive to solving the problems of poor reliability, low security, high cost, and low efficiency in centralized organizations [10, 11].

Similar to cryptocurrencies, many entities (nodes, gateways, and users) in an IoT system do not necessarily trust each other when performing transactions. As a distributed ledger technology, blockchain can fulfill the security requirements of IoT applications. Blockchain technology uses public-key cryptography to record transactions between multiple parties without central coordination. The peer-topeer networks established using blockchain can reduce the risks of single-point failures commonly faced by IoT applications [12, 13]. Blockchain can also enrich IoT applications by keeping transaction records permanently in a verifiable way, and IoT information for which the blockchain provides trusted distributed authentication and authorization is integral and traceable. Therefore, the use of blockchain can strengthen the reliability of IoT applications. Blockchains can use pseudonyms without the need for any identity information to preserve anonymity and privacy [14]. This is attractive for many IoT applications that need to maintain confidential identities and privacy. To sum up, blockchain-based IoT applications would represent a key revolution to solve security, privacy, and reliability problems related to the IoT paradigm.

With the increased focus on the field of blockchain for IoT (BIoT), a large number of studies have emerged. As an objective criterion for evaluating the research performance in a field, bibliometric analyses have become important tools for assessing scholarly quality and productivity $[15,16]$. The bibliometric analysis may examine an array of different objects and have been used in different disciplines [17-20]. Compared to other types of analyses, there are numerous advantages in using bibliometrics: (1) bibliometrics can be used to analyze the number of publications and citations to find influential authors, journals, institutions, and countries efficiently [21]. (2) With the aid of visualization techniques (e.g., VOSviewer software), bibliometrics can be used to analyze information more intuitively by constructing network connections of authors, journals, institutions, countries, and keywords [22]. (3) Bibliometrics can be used to find the research hotspots and foresee future research undertakings [23].

In recent years, bibliometric methods are being increasingly applied to the fields of blockchain and IoT. For example, Firdaus et al. [24] conducted a bibliometric analysis of the blockchain which exhibited the exploration of an in-depth research pattern in this area. Dabbagh et al. [25] conducted a bibliometric study of the blockchain literature with the aim of systematically analyzing all the blockchain research papers that have been indexed by WoS Core Collection. Muessigmann et al. [26] provided a structured bibliometric analysis of blockchain technology in logistics and supply chain management. Nobre and Tavares [27] performed a bibliometric literature review from the Scopus Database focusing on the application of big data/IoT on the context of circular economy. Bouzembrak et al. [28] reviewed the use of IoT technology in food safety using the bibliometric networks. However, there is still a lack of research on BIoT using bibliometric methods. For this reason, in this paper we apply bibliometric methods to sort out the scientific literature of the BIoT domain in recent years and explore publication and citation details, research hotspots, and collaborations among authors, institutions, and countries. More specifically, the present article intends to answer the following questions: which publications and issues in BIoT-related fields have received high attention? Which authors, institutions, countries, and journals significantly contribute to the research domain? Which authors, institutions, and countries are involved in collaborative works? Which journals are closely linked? Which keywords are the hot issues of BIoT research? What are the future research trends for BIoT?

In this study, we aim to provide a holistic vision of the research trends, the most concerned topic and existing research gaps in the field of BIoT. The key contributions of this article lie in two main areas: on the one hand, based on the keyword co-occurrence analysis, the study explores the hot issues in the BIoT field. On the other hand, the study predicts the future research trends in the BIoT field using text mining technology. This study will provide researchers with a greater understanding of the research hotspots and trends, which will be helpful to the researchers for their future research planning.

The remainder of the paper is arranged as follows. In Section 2, we describe the data sources and methods of the study. In Section 3, we demonstrate the results in detail. Section 4 presents hot issues, research trends, and limitations. Section 5 summarizes the key conclusions of the study.

\section{Data and Methods}

The data collected in this study are based on the retrieval of the SCIE and SSCI databases in WoS Core Collection. One of the basic tenets of this content selection for the WoS Core Collection is Garfield's Law of Concentration which postulates that a relatively small core of journals (10-20 percent) account for the bulk (80-90 percent) of what is cited by all published literature, and that these core journals are frequently cited across a wide range of disciplines [29]. Therefore, we mainly analyze the most influential papers about the topic of the BIoT, which are based on the SCIE and SSCI databases. These two databases cover more scientific and authoritative publications than others. For this study, we selected the "topic" search field, and the search terms were 
("blockchain *" and "IoT") or ("blockchain *" and "Internet of things"). The literature searching covered "articles" and "reviews" published during 2016-2020. A total of 917 documents were obtained. Many papers did not include search terms in their title, abstract, and keywords, and these articles were inappropriate to be included in this study. Therefore, we manually screened 917 documents, eliminating articles that did not contain the search terms in the title, abstract, and keywords, and finally obtained 802 documents.

Because no individual bibliometric indicator can account for all aspects of research performance, the collected data from the SCIE and SSCI databases were analyzed using several bibliometric indicators, including the total number of publications (TP), total citations (TC), the percentage of total publications (\%/802), the average citations per paper (AC), and $h$-index. The indicator TP provides information about the number of publications which is necessary for the analysis of productive authors, institutions, and countries. TC is a wellestablished, purely citation-based metric for measuring the quality of scientific papers. It helps to acknowledge and trace the original source and authorship of a concept or an idea. The $\mathrm{AC}$ indicator reflects average citations per paper, and it is useful in performance evaluation and comparative studies [30]. According to [31], the $h$-index can be defined as follows: A researcher has an $h$-index if they have at least $h$ publications for which they have received at least $h$ citations. Therefore, the h-index combines TP and TC in one index [32].

As an important research topic in the field of bibliometrics, bibliometric mapping is a network visualization that reflects the development process and structural relationship of scientific knowledge using the dual attributes of "graph" and "genealogy" $[33,34]$. In recent years, it has become a commonly used method to explore the development of a certain field. Apart from elementary statistics and standard visualization graphs, we utilized the VOSviewer software to prepare a network visualization of the bibliographic coupling of authors, co-authorship of institutions and countries, co-citation of journals, and co-occurrence of keywords. VOSviewer is a software tool which has been developed for constructing graphs based on bibliographic data and visualizing bibliometric maps [35]. It also offers text mining functionality that can be used to construct and visualize cooccurrence networks of important terms extracted from a body of scientific literature [36].

VOSviewer constructs a bibliometric mapping based on a co-occurrence matrix. The construction of the bibliometric mapping consists of three steps: firstly, the similarity matrix is calculated based on the co-occurrence matrix. Secondly, the visualization technique is applied to the similarity matrix to construct a bibliometric mapping. Finally, it is the transformation, rotation, and mapping $[35,37]$.

VOSviewer uses the strength of association to measure similarity. The similarity $s_{i j}$ between individual $i$ and individual $j$ can be expressed by the following formula:

$$
s_{i j}=\frac{c_{i j}}{w_{i} w_{j}},
$$

where $c_{i j}$ represents the number of co-occurrences of the individual $i$ and the individual $j$, and $w_{i}$ and $w_{j}$ represent the number of respective occurrences of the individual $i$ and the individual $j$.

The core idea of the similarity visualization technique is to minimize the weighted square sum of the Euclidean distance. The higher the similarity between the two individuals, the higher the weight of the square sum. To avoid that all individuals are in the same position, a constraint is given that the average distance between two individuals is equal to one. We can represent the objective function and constraints as the following mathematical model:

$$
\begin{aligned}
& \text { Min } V\left(x_{1} \ldots x_{n}\right)=\sum_{i<j} s_{i j}\left\|x_{i}-x_{j}\right\|^{2}, \\
& \text { s.t. } \frac{2}{n(n-1)} \sum_{i<j}\left\|x_{i}-x_{j}\right\|=1,
\end{aligned}
$$

wherein $x_{i}=\left(x_{i 1}, x_{i 2}\right)$ represents the position of the individual $i$ in the two-dimensional map and $\|\bullet\|$ indicates the Euclidean norm.

The optimization problem above has no unique global optimal solution. It needs to solve this problem in three steps: the first step is to transform the solution centered on the origin; the second step is to use the principal component analysis method to maximize the variance of the solution in the horizontal direction by rotation. Thirdly, if the median of $x_{11}, \ldots, x_{\mathrm{n} 1}$ is greater than 0 , the solution is mapping to the vertical axis; if the median of $x_{12}, \ldots, x_{\mathrm{n} 2}$ is greater than 0 , the solution is mapping to the horizontal axis. These three transformations ensure that the VOSviewer produces consistent results.

Items and links together constitute a network, where items are represented by nodes whose size signifies the weights of the items. The distance between two nodes approximately reflects the relatedness between two items; a shorter distance generally presents a stronger relation. Items may be grouped into clusters, whose nodes all bear the same color. Links can exist for any pair of items; a link reveals a relation between two items, and the stronger the link between two items, the thicker the line between two nodes that is used to display the link in the visualization. The link strength (LS) indicates the number of links of a node with other nodes. The total link strength (TLS) represents the sum of the link strengths of this node over all other nodes [23].

\section{Results and Discussion}

3.1. State of the Art in BIoT Research. Using the above method, we retrieved 802 documents from the WoS Core Collection database from 2016 to 2020. The first article on BIoT was published in 2016. The annual change trends of TP are shown in Figure 1. It can be seen that the annual number of publications showed an overall upward trend in the period from 2016 to 2020 . Out of a total of 802 publications, the breakdown for each year from 2016 to 2020 was 2, 6, 64, 236, and 494, respectively. After 2018, there was a 


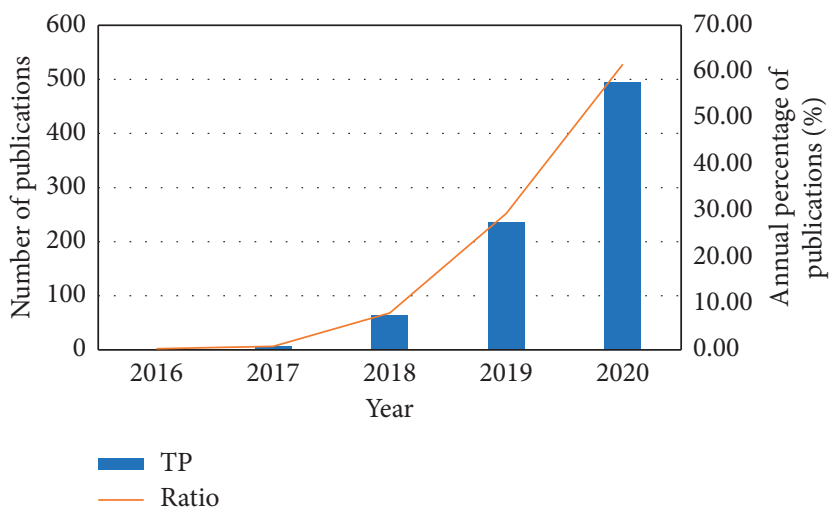

Figure 1: Annual number of total publications.

remarkable increase in the number of BIoT studies. 2020 was the most productive year with 494 publications, i.e., $61.6 \%$ of the total 802 papers, indicating that the research on BIoT is attracting more and more scholars' attention and is becoming a hot research topic.

Table 1 presents the title, author's name, year of publication, and number of total citations of the top 10 mostcited articles. Except for two articles from 2016 and 2019, all other papers were published in 2018 . The top 10 articles from Table 1 discuss the state of the art of different themes of BIoT research. Highly cited articles may affect their respective research subdomains, thereby setting the scope of future research efforts.

The article titled "Blockchains and Smart Contracts for the Internet of Things" [13] ranked first with 1003 total citations. In that article, K. Christidis and M. Devetsikiotis examine whether blockchains are suitable for Internet of Things (IoT) applications. The conclusion is that the blockchain-IoT combination can cause significant transformations across several industries. The paper written by M.A. Khan and K. Salah entitled "IoT security: review, blockchain solutions, and open challenges" [38] is the second influential work in Table 1 . The article analyzes how blockchain can be a key enabler and solve many IoT security problems. The article written by Zheng et al. entitled "blockchain challenges and opportunities: a survey" [39] is the third in the list, cited 407 times. The authors believe that blockchain can help in improving the IoT sector such as e-business, safety, and privacy.

3.2. Analysis of Leading Authors. A total of 2676 authors participated in BIoT research. Table 2 enumerates the top 10 authors with the highest number of publications. J.H. Park from Seoul National University of Science and Technology published 18 papers on BIoT, becoming the most prolific author. He is Associate Editor/Editor of 14 international journals. The main areas of his research include IoT, Human-Centric Ubiquitous Computing, Information Security, Digital Forensics, Vehicular Cloud Computing, Multimedia Computing, etc. M. Guizani from Qatar University is listed in the second position with 16 items. He is currently the Editor-in-Chief of the IEEE Network Magazine. His research interests include wireless communications and mobile computing, computer networks, mobile cloud computing, security, and smart grid. Y. Zhang from University of Oslo ranks third with 14 papers, followed by X.J. Du $(\mathrm{TP}=13)$ from Temple University, and K-K.R. Choo $(\mathrm{TP}=12)$ from University of Texas at San Antonio. The top 10 high-yielding scholars contributed 119 papers, accounting for $14.84 \%$ of all publications.

Highly cited authors are considered to be the world's most influential researchers, because they usually grasp the research hotspots and cutting-edge trends in certain subject areas. Table 3 presents the top 10 authors with the highest number of citations. It can be seen that K. Christidis and M. Devetsikiotis, two scholars from North Carolina State University with 1003 citations, are the most-cited authors, followed by Z.B. Zheng of Sun Yat Sen University, J.H. Park of Seoul National University of Science and Technology, and H.N. Dai of Macau University of Science and Technology, cited 536, 533, and 504 times, respectively. In addition, from the perspective of the country distribution of most influential authors, China, the United States, and Korea dominate the research in the field of BIoT.

Bibliographic coupling analysis is an important tool for establishing the connections among authors who cite the same publications [40]. The more often two researchers cite the same publications, the stronger their relatedness [41]. Figure 2 presents a visualization of the authors' bibliographic coupling network. Only authors with more than 5 articles are labeled. Of the 2676 authors, 39 meet this threshold. The node size represents the number of publications while the thickness of the connecting lines represents the link strength. As shown in Figure 2, the authors are grouped into 7 clusters represented by 7 colors. F.R. Yu (TLS =2320), M. Guizani $(\mathrm{TLS}=2760), \quad \mathrm{N}$. Kumar $\quad(\mathrm{TLS}=3795), \quad \mathrm{M}$. Imran $(T L S=1434)$, J.H. Park $(T L S=3045)$, W.J. Li $(T L S=1032)$, and T.M. Fernandez-Carames $($ TLS $=3781$ ) are authors with the greatest bibliographic couplings in the red, green, blue, yellow, purple, turquoise, and orange clusters, respectively. Overall, N. Kumar (TLS = 3795) is the most bibliographically coupled author, followed by T.M. Fernandez-Carames $(\mathrm{TLS}=3781)$ and K-K.R. Choo (TLS = 3505). Most notably, N. Kumar had awarded the best research paper awards from IEEE ICC 2018 and IEEE Systems Journal 2018. At present, 
TABLE 1: List of top 10 influential papers.

\begin{tabular}{|c|c|c|c|c|}
\hline Rank & Title & Author(s) & Year & TC \\
\hline 1 & Blockchains and smart contracts for the internet of things & Christidis, K.; Devetsikiotis, M. & 2016 & 1003 \\
\hline 2 & IoT security: review, blockchain solutions, and open challenges & Khan, M.A.; Salah, K. & 2018 & 430 \\
\hline 3 & Blockchain challenges and opportunities: a survey & $\begin{array}{l}\text { Zheng, Z.B.; Xie, S.A.; Dai, H.N.; Chen, X.P.; } \\
\text { Wang, H.M. }\end{array}$ & 2018 & 407 \\
\hline 4 & $\begin{array}{c}\text { On blockchain and its integration with loT. Challenges and } \\
\text { opportunities }\end{array}$ & $\begin{array}{c}\text { Reyna, A.; Martin, C; Chen, J.; Soler, E.; Diaz, } \\
\text { M. }\end{array}$ & 2018 & 255 \\
\hline 5 & Blockchain's roles in meeting key supply chain management objectives & Kshetri, N. & 2018 & 251 \\
\hline 6 & $\begin{array}{l}\text { Blockchain meets IoT: an architecture for scalable access management } \\
\text { in IoT }\end{array}$ & Novo, $\mathrm{O}$. & 2018 & 236 \\
\hline 7 & $\begin{array}{c}\text { Consortium blockchain for secure energy trading in industrial internet } \\
\text { of things }\end{array}$ & $\begin{array}{c}\text { Li, Z.T.; Kang, J.W.; Yu, R.; Ye, D.D.; Deng, } \\
\text { Q.Y.; Zhang, Y. }\end{array}$ & 2018 & 229 \\
\hline 8 & $\begin{array}{c}\text { Blockchain technology in the energy sector: a systematic review of } \\
\text { challenges and opportunities }\end{array}$ & Andoni, M.; Robu, V.; Flynn, D. & 2019 & 214 \\
\hline 9 & A review on the use of blockchain for the Internet of things & Fernandez-Carames, T.M.; Fraga-Lamas, P. & 2018 & 188 \\
\hline 10 & $\begin{array}{l}\text { A software defined fog node based distributed blockchain cloud } \\
\text { architecture for IoT }\end{array}$ & Sharma, P.K.; Chen, M.Y.; Park, J.H. & 2018 & 178 \\
\hline
\end{tabular}

TABle 2: Top 10 most productive authors.

\begin{tabular}{lcr}
\hline Author & Institution & Country \\
\hline Park, J.H. & Seoul National University of Science and Technology & South Korea \\
Guizani, M. & Qatar University & Qater \\
Zhang, Y. & University of Oslo & Norway \\
Du, X.J. & Temple University & USA \\
Choo, K-K.R. & University of Texas at San Antonio & 14 \\
Kumar, N. & Thapar Institute of Engineering and Technology & 13 \\
Sharma, P.K. & Seoul National University of Science and Technology & 12 \\
Fernandez-Carames, T.M. & Universidade da Coruna & India \\
Fraga-Lamas, P. & Universidade da Coruna & South Korea \\
Li, H. & Old Dominion University & Spain \\
\hline
\end{tabular}

TABLE 3: Top 10 most influential authors.

\begin{tabular}{lcc}
\hline Author & Institution & Country \\
\hline Christidis, K. & North Carolina State University & USA \\
Devetsikiotis, M. & North Carolina State University & USA \\
Zheng, Z.B. & Sun Yat Sen University & China \\
Park, J.H. & Seoul National University of Science and Technology & South Korea \\
Dai, H.N. & Macau University of Science and Technology & China \\
Salah, K. & Khalifa University of Science and Technology & 536 \\
Khan, M.A. & Bahauddin Zakariya University & 533 \\
Zhang, Y. & University of Oslo & 504 \\
Sharma, P.K. & Seoul National University of Science and Technology & 473 \\
Xie, S.A. & Sun Yat Sen University & Pakistan Emirates \\
\hline
\end{tabular}

he is leading the Sustainable Practices for the Internet of Energy and Security research group, where team members are working on the latest cutting-edge technologies. The cooperation network centered on N. Kumar represents the forefront of research in this field. By pairing authors, the strongest link strength is found between T.M. FernandezCarames and P. Fraga-Lamas $(L S=1917)$ affiliated with Universidade da Coruna of Spain, followed by N. Kumar and S. Tanwar $(L S=743)$ and M. Guizani and X.J. Du $(L S=558)$.
3.3. Analysis of Dominant Institutions. A total of 1073 institutions are involved in research related to BIoT. Table 4 lists the top 10 institutional contributors to BIoT research and ranks them according to the number of publications. In addition, the table captures other relevant information including country, percentage of total publications, number of average citations, and $h$-index.

The Beijing University of Posts and Telecommunications $(38,4.74 \%)$ is the largest provider of BIoT research. The 


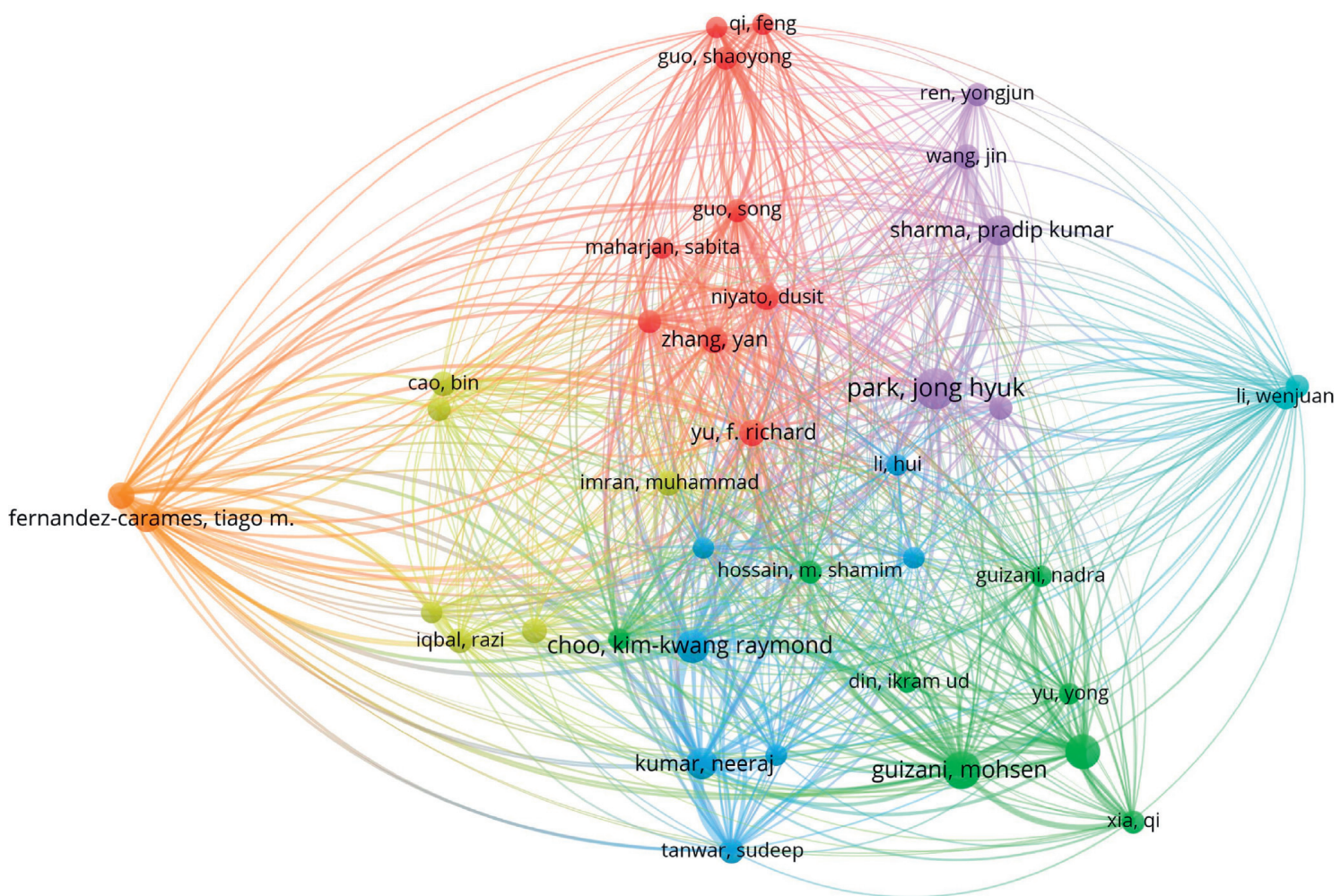

FIgURE 2: Bibliographic coupling network of authors.

TABLE 4: Top 10 most productive institutions.

\begin{tabular}{|c|c|c|c|c|c|}
\hline Institution & Country & $\mathrm{TP}$ & $\% 802$ & $\mathrm{AC}$ & $h$-index \\
\hline Beijing University of Posts and Telecommunications & China & 38 & 4.74 & 18.87 & 11 \\
\hline King Saud University & Saudi Arabia & 26 & 3.24 & 18.73 & 13 \\
\hline University of Electronic Science and Technology of China & China & 25 & 3.12 & 12.76 & 10 \\
\hline Chinese Academy of Sciences & China & 23 & 2.87 & 13 & 8 \\
\hline Seoul National University of Science and Technology & South Korea & 20 & 2.49 & 26.75 & 8 \\
\hline Xidian University & China & 20 & 2.49 & 11.15 & 8 \\
\hline Pennsylvania Commonwealth System of Higher Education & USA & 18 & 2.24 & 9.5 & 8 \\
\hline Tsinghua University & China & 18 & 2.24 & 16.11 & 6 \\
\hline Comsats University Islamabad (CUI) & Pakistan & 17 & 2.12 & 9.82 & 6 \\
\hline University System of Georgia & USA & 16 & 2 & 10.13 & 7 \\
\hline
\end{tabular}

second position is held by the King Saud University (26, $3.24 \%$ ), followed by University of Electronic Science and Technology $(25,3.12 \%)$ and the Chinese Academy of Sciences $(23,2.87 \%)$. However, from the perspective of average citations, the Seoul National University of Science and Technology leads Table 3 with 26.75 average citations, followed by Beijing University of Posts and Telecommunications with 18.87 average citations and King Saud University with 18.73 average citations. In terms of $h$-index, the King Saud University tops Table 4, followed by Beijing University of Posts and Telecommunications and University of Electronic Science and Technology of China. It is worth noting that of the 10 most productive institutions, five are from China, two institutions are from the United States, one from South Korea, one from Saudi Arabia, and one from Pakistan. This indicates with no doubt that in terms of total publications, Chinese institutions play an important role in global BIoT research. The 10 most productive institutions have contributed 221 papers, accounting for $27.56 \%$ of all publications in this field.

Figure 3 shows the ten most influential institutions and ranks them according to the number of total citations. The North Carolina State University, with 1003 citations, leads Figure 3 followed by Beijing University of Posts and Telecommunications (717), Sun Yat Sen University (566), and Macau University of Science and Technology (504). Note the significant contribution of the two authors: K. Christidis and M. Devetsikiotis from North Carolina State University, who have contributed 1003 citations. In the top ten places, there are five institutions from China, one institution from the USA, one institution from Saudi Arabia, one institution from Pakistan, one institution from United Arab Emirates, 


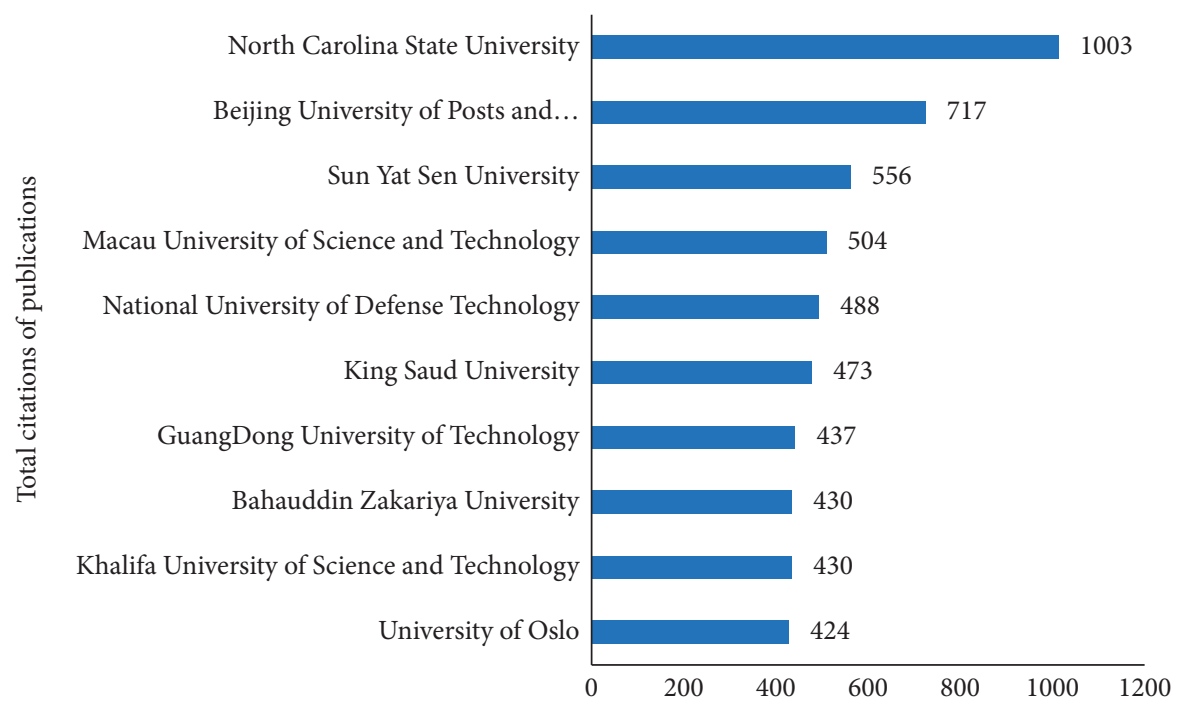

FIgURE 3: Top 10 highly cited institutions.

and one institution from Norway. It is worth noting that China is in the leading position in terms of institutional influence.

As an important aspect of bibliometric studies, co-authorship analysis aims to explore the level of research collaborative strength in a specific field [23]. Figure 4 depicts visualization of the institutions' co-authorship network, where the minimum number of documents for inclusion in the figure is 5. Among the 1073 institutions, 84 meet the threshold. The size of the circle represents the number of publications. The connection between the two institutions represents a cooperative relationship. Figure 4 clusters the institutions into ten groups coded with different colors. Beijing University of Posts and Telecommunications (TLS $=41$ ) is at the center of cooperation and has the strongest co-authorship network, followed by the University of Electronic Science and Technology of China (TLS = 37) and King Saud University (TLS $=31$ ). Considering the links between the two institutions, the strongest co-authorship collaboration exists between Temple University and Qatar University $(\mathrm{LS}=8)$ followed by Temple University and University of Electronic Science and Technology of China $(\mathrm{LS}=6)$ and by Chinese Academy of Sciences and University of Chinese Academy of Sciences (TLS =6). The figure reveals that although institutions in geographic proximity are more closely linked, geographical advantage is not the main factor influencing interinstitutional cooperation as international academic exchanges become more frequent.

3.4. Analysis of Key Countries/Regions. A total of 72 countries are active in the field of BIoT research. Figure 5 displays the top 10 countries/regions in the world in terms of number of publications and citations. China published the highest number of literature items (337 articles), followed by the United States (141), South Korea (100), India (77), and England (71). China's literature items received a total of 4306 citations as the highest citation number of all countries, followed by the USA (3200), South Korea (1339), England
(968), and India (954). As can be seen, the number of publications and citations in China and the United States is much higher than in other countries. This reveals that China and the USA have been at the forefront of the world in BIoT research and are likely to lead the development of global BIoT in the next decade.

Co-authorship collaboration among countries is shown in Figure 6, which displays countries with more than five articles. Of the 72 countries, 37 meet the inclusion criteria. The countries are grouped into 6 clusters of different colors. China (TLS $=294$ ) is at the center of collaboration and has the most cooperation with other countries. The countries that have close cooperation with China mainly include the USA (LS = 69), England ( $L S=22)$, Australia $(L S=19)$, and Canada $(L S=17)$. The USA (TLS $=171)$ is the second largest co-authorship country, and its main partners are China $(\mathrm{LS}=69)$, England (LS=27), Australia (LS = 26), and Canada $(\mathrm{LS}=18)$. This indicates that collaboration between countries can increase the amount of publications in comparison to publications from a single country. In addition, it is worth mentioning that the collaboration between China and the United States is the most intensive.

3.5. Analysis of Popular Journals. A total of 164 journals are pertinent to the field of BIoT research. Table 5 depicts the basic information of the top 10 most productive journals. Among them, IEEE Access (110) had the greatest number of publications, accounting for $13.71 \%$. It was followed by IEEE Internet of Things Journal (74) and Sensors (65), accounting for $9.23 \%$ and $8.1 \%$, respectively. IEEE Access had not only published most articles but also had the largest number of total citations (2578). However, from the perspective of average citation, Future Generation Computer Systems had the highest number of average citations (36.71), followed by IEEE Transactions on Industrial Informatics (25.84) and IEEE Access (23.44). In terms of the $h$-index, IEEE Access again led Table 5 with the highest $h$-index (21), while IEEE Internet of Things Journal (17) obtained the second position 


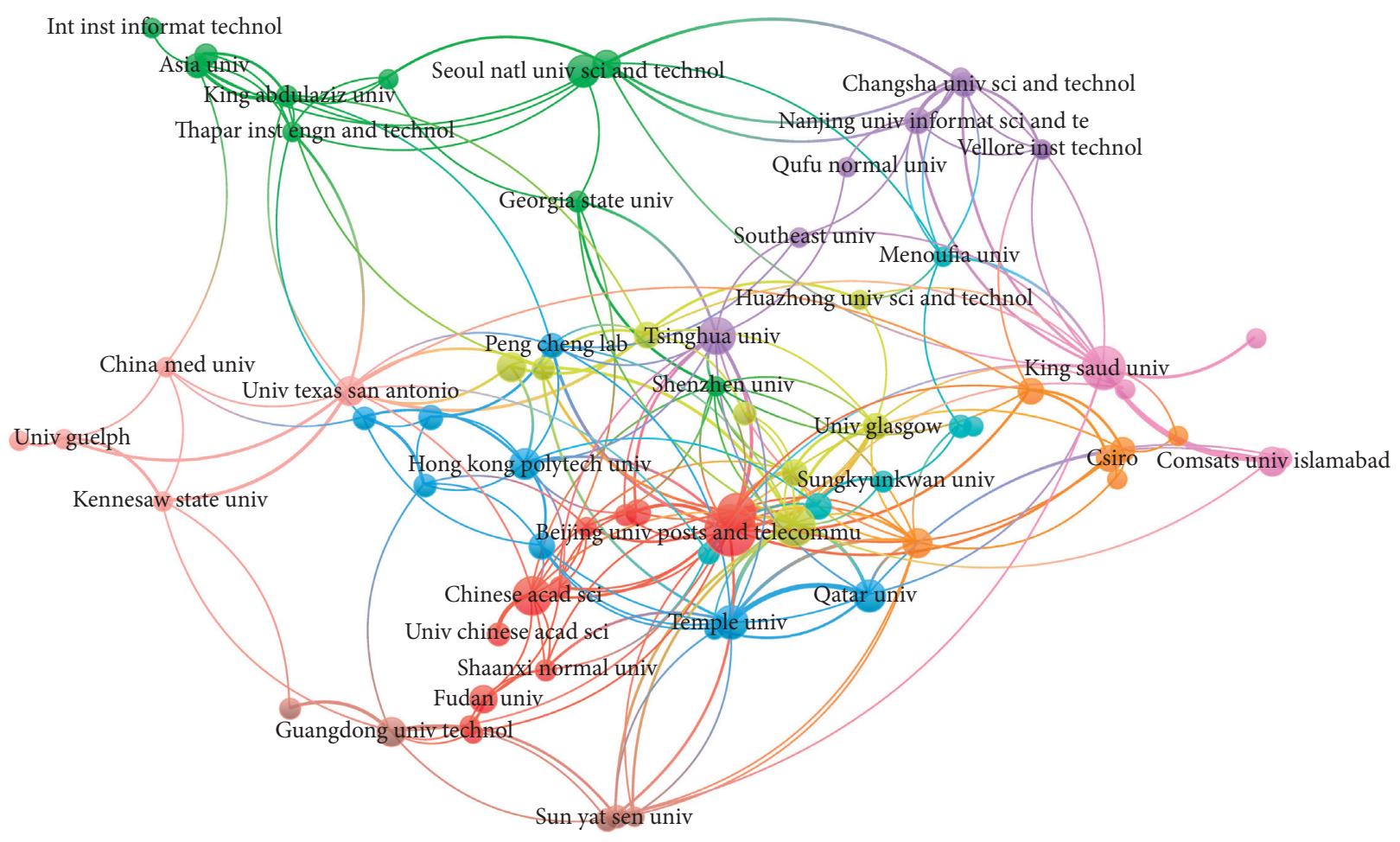

FIgURE 4: Co-authorship network of institutions.

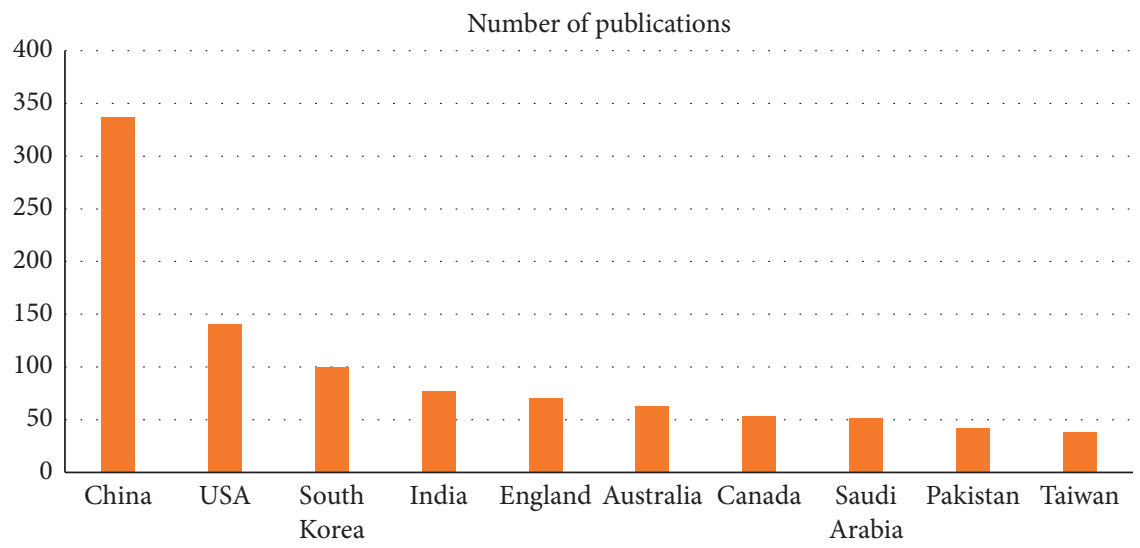

(a)

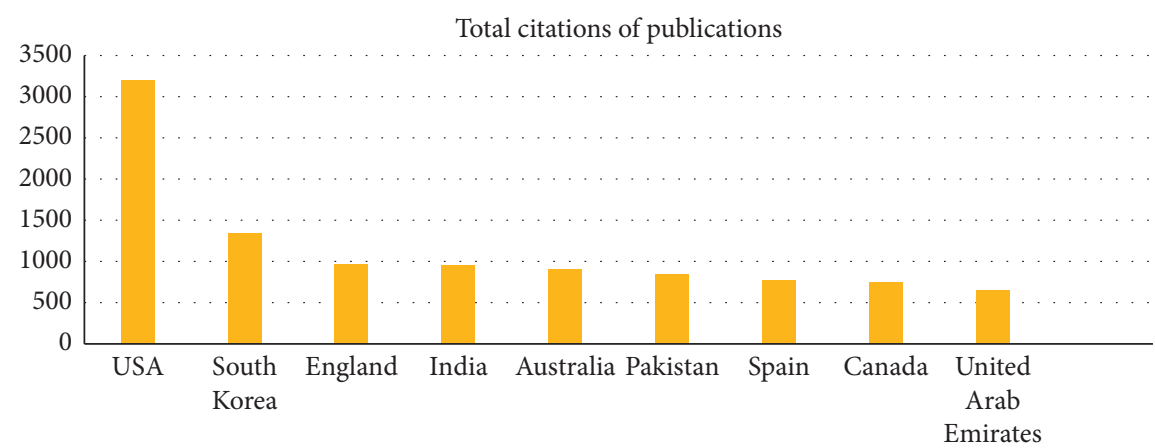

(b)

Figure 5: (a) Number of Publications; (b) total citations of publications. 


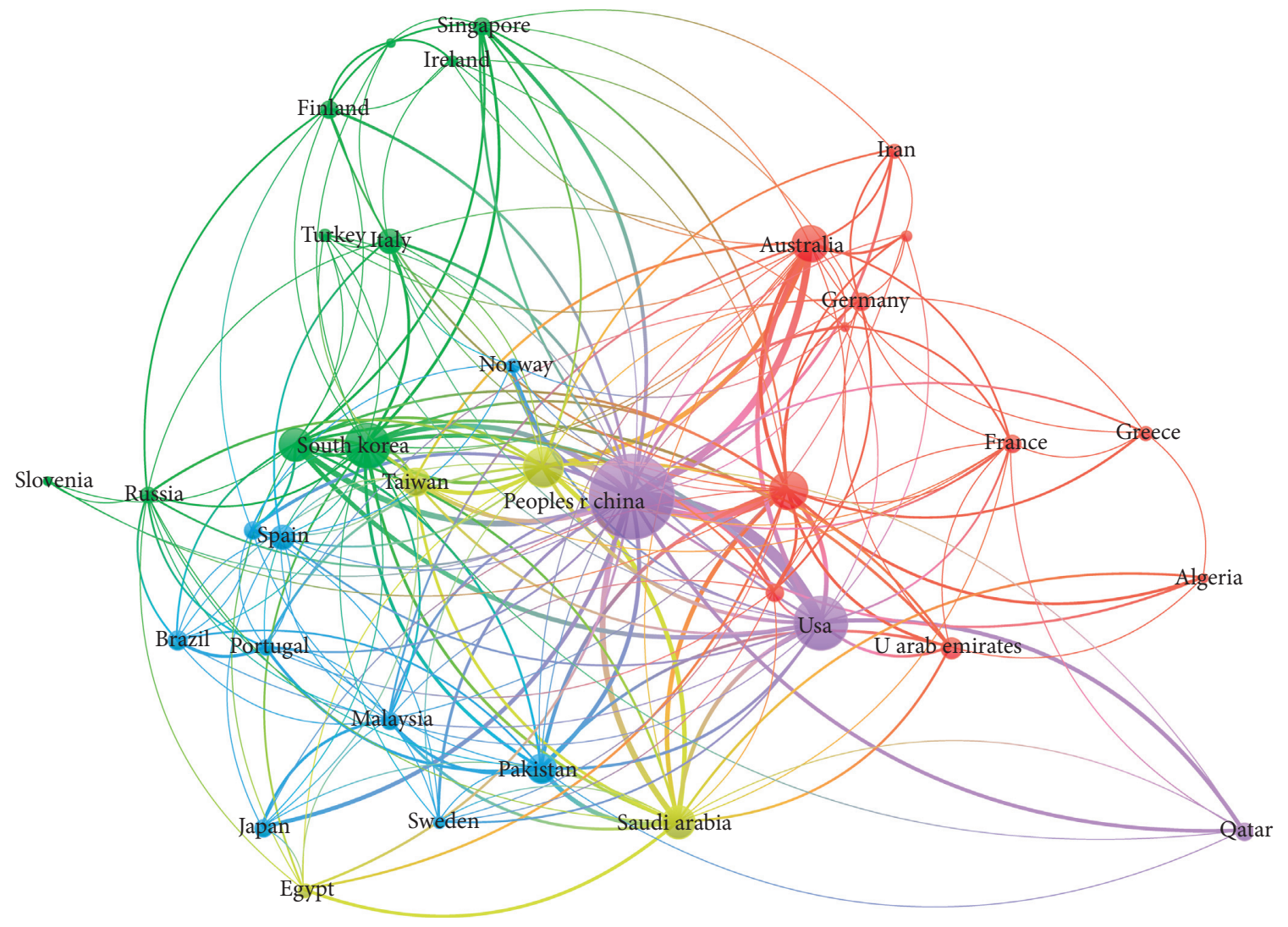

Figure 6: Co-authorship network of countries.

TABle 5: Top 10 most productive journals.

\begin{tabular}{|c|c|c|c|c|c|}
\hline Journal & $\mathrm{TP}$ & $\% 802$ & TC & $\mathrm{AC}$ & $h$-index \\
\hline IEEE Access & 110 & 13.71 & 2578 & 23.44 & 21 \\
\hline IEEE Internet of Things Journal & 74 & 9.23 & 1152 & 15.57 & 17 \\
\hline Sensors & 65 & 8.1 & 484 & 13.05 & 13 \\
\hline IEEE Transactions on Industrial Informatics & 37 & 4.61 & 956 & 25.84 & 16 \\
\hline Future Generation Computer Systems & 31 & 3.87 & 1138 & 36.71 & 13 \\
\hline Electronics & 27 & 3.37 & 116 & 4.3 & 6 \\
\hline IEEE Network & 24 & 2.99 & 203 & 8.46 & 9 \\
\hline Applied Sciences-Basel & 17 & 2.12 & 84 & 4.94 & 6 \\
\hline Sustainability & 15 & 1.87 & 80 & 5.33 & 4 \\
\hline Transactions on Emerging Telecommunications Technologies & 14 & 1.75 & 47 & 3.36 & 4 \\
\hline
\end{tabular}

followed by IEEE Transactions on Industrial Informatics (16).

We now turn to the analysis of co-citation network of journals. Co-citation of journals occurs when two documents of two different journals are cited from the same third publication [42]. The more often two journals are cited in the same document, the stronger their relatedness. In this study, the co-citation analysis of journals with a minimum of 50 citations is considered. Only 100 journals out of 13320 cocited sources meet this criterion. As shown in Figure 7, the journals are grouped into 4 clusters using 4 colors. IEEE Access tops Figure 6 with 62676 total link strengths, followed by IEEE Internet of Things Journal (TLS $=43272$ ) and Lecture Notes in Computer Science (TLS=29719). According to the journal collaboration network, the most frequent interactions are between IEEE Access and IEEE Internet of Things Journal $(\mathrm{LS}=6736)$ followed by IEEE Access and Future Generation Computer Systems $(\mathrm{LS}=3737)$, and by IEEE Access and IEEE Communications Surveys and Tutorials (LS=3372).

\section{Hot Issues, Research Trends, and Limitations}

4.1. Hot Issues. Keyword co-occurrence can provide insights into the research hotspots in a certain discipline, revealing the research frontiers of the subject. In this study, the cooccurrence of author keywords is analyzed. The minimum number of occurrences of a keyword is set to 5, and 102 out of 1907 keywords meet this threshold. In Figure 8, these keywords are grouped into 8 colored clusters according to 


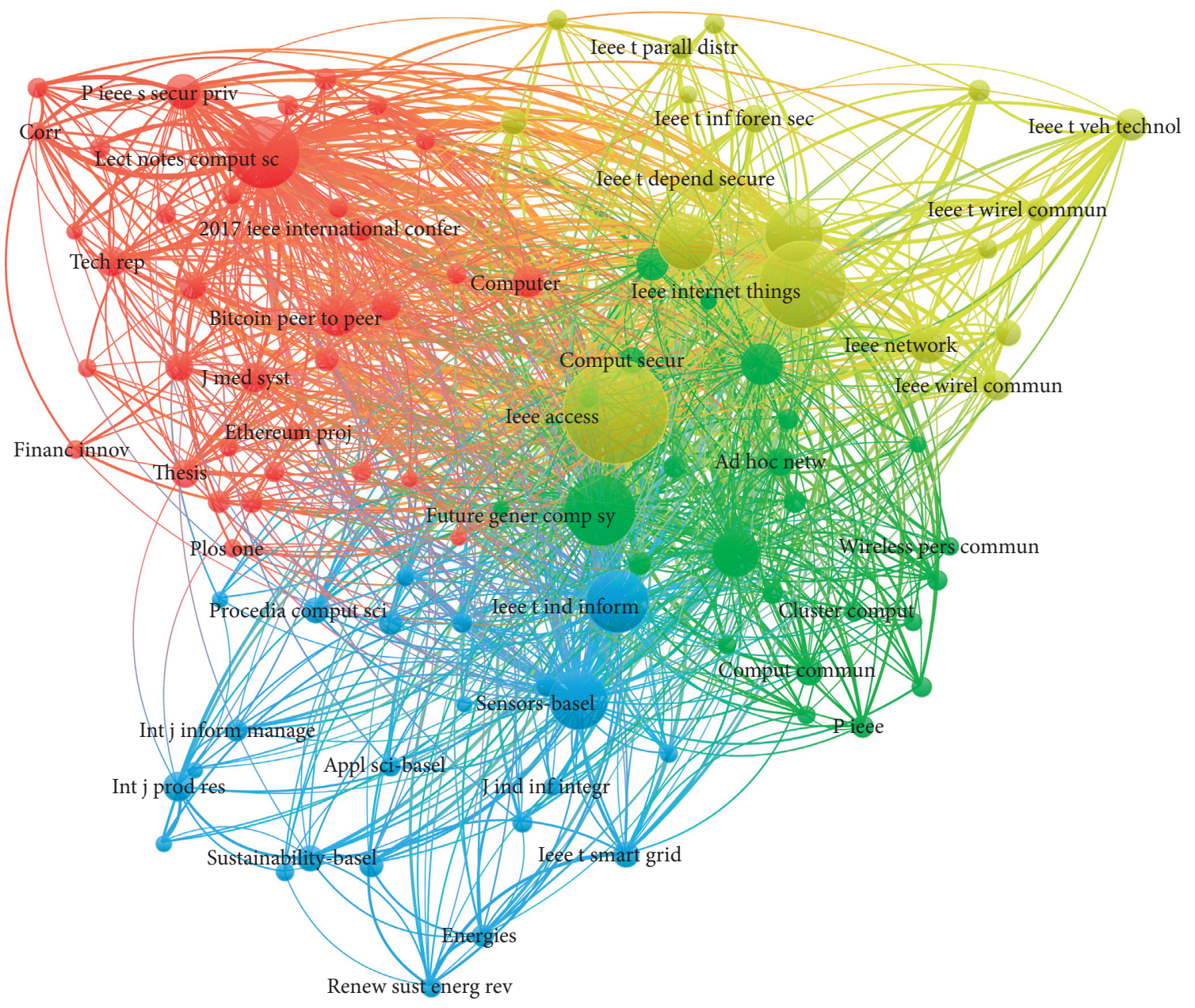

FIgURE 7: Co-citation network of journals.

the similarity of research fields. The high-frequency keywords reflect relevant research hotspots and tendencies. The 15 most frequent keywords are "blockchain" (582 occurrences), "Internet of things" (462 occurrences), "security" (119 occurrences), "smart contract" (99 occurrences), “edge computing" (61 occurrences), "industrial Internet of things" (59 occurrences), "privacy" (51 occurrences), "cloud computing" (42 occurrences), "fog computing" (37 occurrences), "access control" (35 occurrences), "smart city" (31), "industry 4.0" (30 occurrences), "authentication" (28 occurrences), "peer-to-peer computing" (25 occurrences), and "bitcoin" (24 occurrences). It is worth mentioning that the strongest co-occurrence networks are between "blockchain" and "Internet of things" (LS = 198), followed by between "blockchain" and "security" $(\mathrm{LS}=107)$. According to Table 6, the potentially hot issues around BIoT research can be categorized into four aspects: security issues, core technologies, application dimensions, and transaction processes.

4.1.1. Security Issues. Since a typical IoT deployment contains heterogeneous devices with embedded sensors through interconnected networks, it inherits the drawbacks of conventional computer networks related to security vulnerabilities and privacy risks [38]. As a decentralized and distributed ledger technology, blockchain can satisfy the security requirements and promote the development of secure IoT applications, such as anonymous authentication, audit capability, and confidentiality. It highlights the scalability capacity that comes with smart contracts $[10,11]$. Within the blockchain network, smart contracts stored on the blockchain have a unique address, and users trigger a smart contract by sending a transaction to this address. The contractual clauses are then executed independently and automatically. Blockchain can secure communications between devices validated through smart contracts [43]. Smart contracts will enrich the IoT by providing a secure and reliable processing engine which records and manages all their interactions. With smart contracts, blockchain has the potential to improve the security of IoT applications.

One of the key challenges for IoT applications is privacy, mainly due to the large scale and distributed nature of IoT networks. The blockchain can alleviate the problem through advanced cryptography technologies [44]. As an important part of blockchain technology, consensus mechanisms are not only responsible for the integrity of the information contained in the blockchain but also help defend against double-spending attacks [10]. On the other hand, 


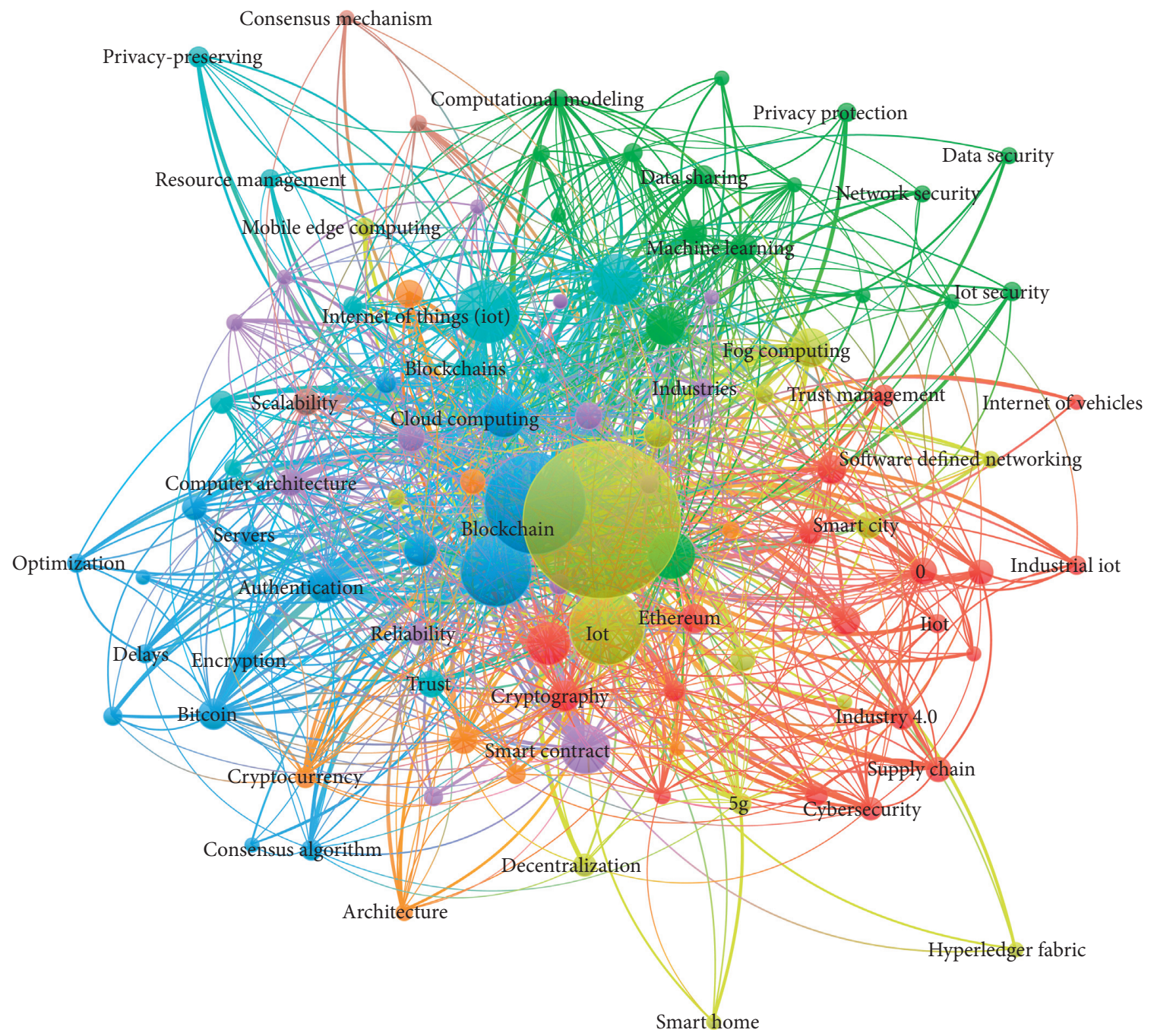

FIgURE 8: Co-occurrence network of author keywords.

TABLE 6: Main research categories in the field of BIoT.

\begin{tabular}{|c|c|}
\hline Category & Representative keywords \\
\hline Security issues & $\begin{array}{r}\text { Security (119), smart contract (99), privacy (51), acces } \\
\text { (16), consensus (16), distributed leds }\end{array}$ \\
\hline Core technologies & $\begin{array}{c}\text { Edge computing (61), cloud computing (42), fog computing (37), peer-to-peer computing (25), computer } \\
\text { architecture (18), machine learning (17), artificial intelligence (17), consensus algorithm (10), computational } \\
\text { modelling (9) }\end{array}$ \\
\hline $\begin{array}{l}\text { Applic } \\
\text { dimens }\end{array}$ & $\begin{array}{l}\text { Industrial Internet of things ( } 59 \text { occurrences), smart city (31), industry } 4.0 \text { ( } 30 \text { occurrences), healthca } \\
\text { chain (14), smart grid (11), } 5 \mathrm{~g}(10) \text {, smart home (6), energy trading (6), Internet of vehic }\end{array}$ \\
\hline Transaction processes & Smart contract (99), bitcoin (24), Ethereum (21), protocols (15), cryptocurrency (10), servers (10), task analysis (9) \\
\hline
\end{tabular}

blockchain-based identity authentication and access control techniques can be leveraged to enhance IoT security. For example, the blockchain is applied to implement permitted access control for devices in the IoT to securely track data management and prevent malicious access [45]. Blockchains can provide trusted distributed authentication and stronger defense against attacks from IoT applications, as the management of certificate data is done on the blockchain, so it is not possible for an attacker to inject fake signatures into the record $[5,12]$.
4.1.2. Core Technologies. The increase in the number of IoT devices has resulted in a large number of IoT terminals. Therefore, a significant portion of computing power at terminals based on blockchain technology would inevitably be consumed. Cloud computing allows for centralized data processing, where the big data generated by distributed sensors are transmitted to a cloud computing data center through the network. These data are centrally processed and stored, leveraging the computational power of the cloud platform. Using cloud computing and big data, blockchain- 
based cloud platforms for IoT device management can adopt more efficient cryptographic algorithms and leverage distributed capabilities to ensure low latency and high throughput in data management [46]. Furthermore, blockchain technology as a distributed ledger and the decentralized edge computing model has also revolutionized the IoT by adding a new layer between cloud computing and IoT devices. Edge computing brings the cloud capabilities closer to the computation tasks. The convergence of blockchain and edge computing paradigms can overcome the security and scalability issues of IoT [47].

The diversification and the rapid increase of IoT devices lead to many identity authentication tasks, causing the users to wait too long. With the development of cloud computing, edge computing, and fog computing, among others, these technologies can not only achieve effective identity authentication but also guarantee the security and reliability of data information. For example, edge computing can improve the capability of data caching and computing. Based on edge computing, the delay-tolerant computing tasks can be offloaded to a closer edge computing server, and then more computing tasks can be selectively accommodated and executed on the local device, edge computing server, or cloud computing server according to the current network states and network environments [48]. Compared with the traditional IoT architecture, this can reduce the end-to-end delay between IoT devices and computing resources, thereby facilitating IoT service provisioning.

4.1.3. Application Dimensions. By providing a secure open communication platform, BIoT can offer many possible applications for smart cities such as smart home, smart management, and smart gadgets [49]. Smart cities can improve the efficiency of resource utilization, optimize city management and services, and make city life easier by exploiting data collection sensors to transmit information. One smart city BIoT application is detailed in [50], where the use of blockchain technology is analyzed in different smart city business models.

The energy sector can also benefit from the application of BIoT. In [51], a prototype implementation using the Ethereum platform, a smart energy grid capable of timely adjustment of the energy demand by enacting the expected energy flexibility levels and validating all demand response protocols is analyzed. In [52], a decentralized privacy-preserving healthcare blockchain application for IoT is proposed. The results showed that IoT application data and transactions are more secure and anonymous over a blockchain-based network. In [53], various mechanisms were illustrated whereby blockchain helps achieve the key supply chain management objectives such as cost, quality, speed, dependability, risk reduction, sustainability, and flexibility. In addition, there are some other application scenarios of BIoT, e.g., industry 4.0 [54], 5G [55], Internet of vehicles [56], global value chains [57], Stock Trade [58], and smart grid [59].
4.1.4. Transaction Processes. Blockchain can accelerate the creation of services and data marketplaces for the IoT ecosystem, where transactions between peers can be carried out without the need for trusted parties. Bitcoin, the first cryptocurrency, is also the most popular application that runs on top of the blockchain platform. In general, the bitcoin blockchain provides a secure payment mechanism to carry out monetary transactions in a fast, cheap, and reliable manner. However, its poor scalability limits its use [3]. Ethereum is currently the most popular blockchain-based platform built for creating smart contracts. It focuses more on running programming codes and a consensus-based virtual machine (Ethereum Virtual Machine). Different from transactions in the bitcoin blockchain, which tracks the transfer of ownership of cryptocurrencies, the Ethereum blockchain performs state transitions under an accountbased system which consists of two types of accounts, externally owned accounts controlled by private keys and contract accounts controlled by codes in contracts $[4,60]$. More importantly, the Ethereum blockchain implements smart contracts, which are computerized transaction protocols that execute the terms of the contract. IoT devices can leverage these capabilities to carry out autonomous transactions through smart contracts. The inclusion of smart contracts makes the potential space for Ethereum blockchain in IoT immense and endless [61]. Note that similar smartcontract blockchain platforms have recently emerged, such as Hyperledger [62], BigchainDB [63], Stellar [64], and Tendermint [65].

4.2. Research Trends. A bibliometric analysis can provide an indication of potential research trends and identify possible fields of future progress and engagement. The purpose of this section is to highlight some priorities for further research using text mining analysis to better understand the research trends in the field of BIoT. The text mining functionality of VOSviewer offers support for creating term maps based on a corpus of documents. A term map is a two-dimensional map in which terms are located in such a way that the distance between two terms indicates the relatedness of the terms, which in turn is determined through the co-occurrences in documents [66]. Figure 9 presents an overlay visualization term map using terms extracted from the title and abstracts of a corpus of academic publications in the field of BIoT. A full counting method has been chosen, and the minimum number of occurrences of a term is set to 35 . Of the 16309 terms, 143 meet this threshold. For each of the 143 terms, a relevance score is calculated. Based on this score, the 86 most relevant terms are selected.

In Figure 9, the color of a term is determined by the score of the term, where by default the colors range from blue (lowest score) to green to yellow (highest score). As can be seen, prominent terms in this area include "edge computing," "algorithm," "cost," "block," "protocol," "performance," "simulation result," "big data," "artificial intelligence," "iiot," "communication," "feasibility," "traceability," "transparency," and so on. Therefore, it is likely that 


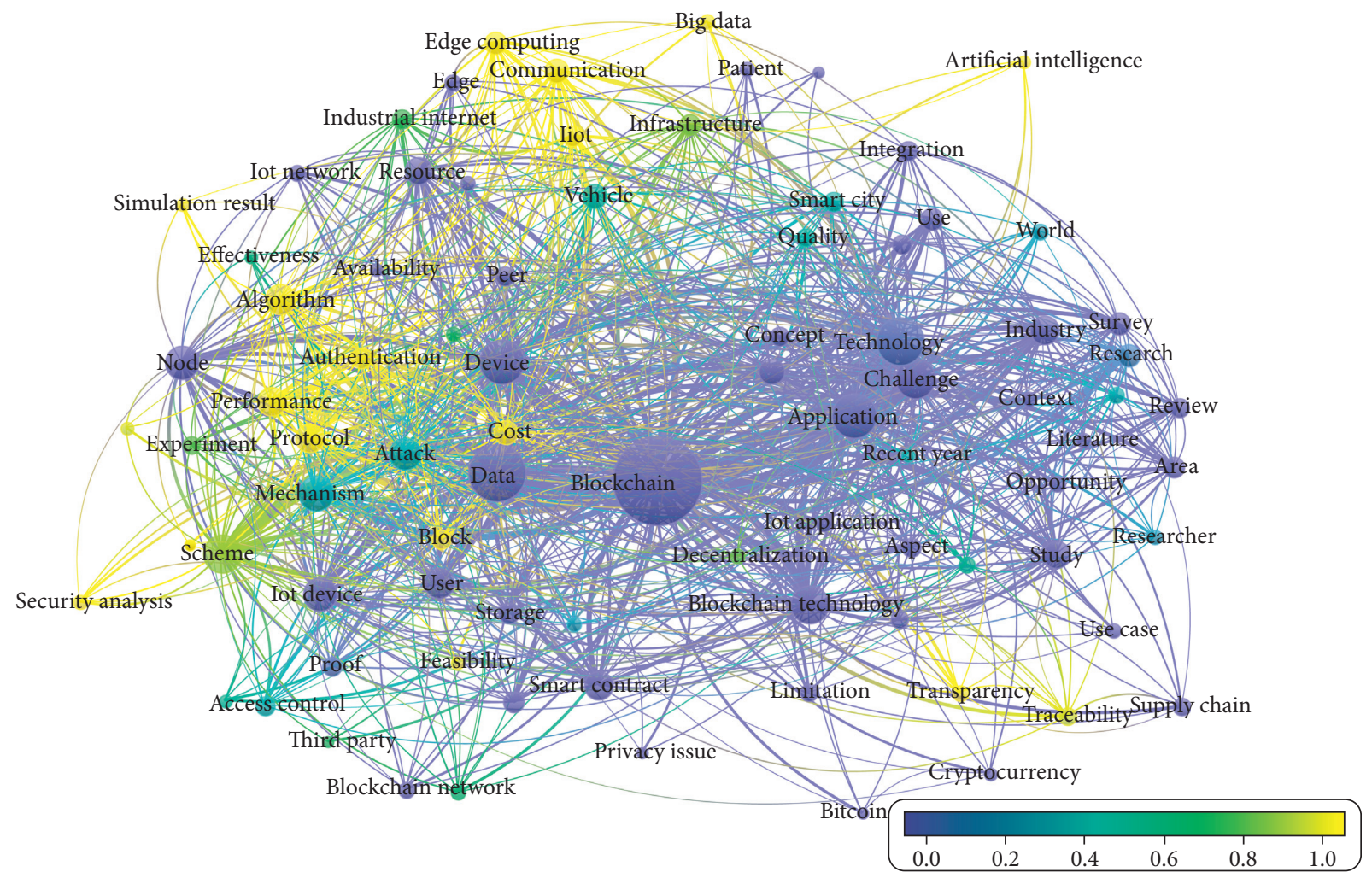

FIgURE 9: Term map based on publications.

future research directions for BloT will focus more on both computing paradigms and key applications.

In terms of computing paradigms, traditional cloud computing is unable to meet the real-time and efficient data processing requirements due to the situations of limited bandwidth and increased network latency. For these reasons, in recent years, cloud computing has evolved towards more complex edge computing and fog computing. Edge computing pertains to data processing at the edge of the network close to the data source. It has gained considerable popularity in academic and industrial circles because of its ability to provide low latency, high bandwidth, and location awareness support for latencysensitive applications [67]. Extensive simulation results with different parameters show that edge computing has more advantages and effectiveness compared with cloud computing [48]. Fog computing, which seamlessly integrates edge devices and cloud resources, is a distributed paradigm that provides cloud-like services to the network edge. It can significantly alleviate the traffic in the core network and potentially accelerate big data processing. Fog computing solves the requirements of widely distributed applications that require low latency $[68,69]$. As discussed, future research will focus more on the performance of fog and edge computing in BIoT applications. Despite the promising benefits and the exciting future of edge and fog computing, there are significant challenges in security and privacy, system integration, and resource management [70]. These challenges will also trigger more research on related topics.
Regarding corresponding BIoT-based applications, future research directions are likely to concentrate on the topics of artificial intelligence, big data, communication, and industrial IoT (IIoT), etc. The convergence of blockchain and artificial intelligence in the IoT network will be an important area of future research and is revolutionizing the smart city network architecture to build sustainable ecosystems [71]. It has the potential to provide real-time costefficient solutions for several applications such as healthcare, smart grid surveillance, smart city monitoring, and border surveillance [72]. As IoT applications become sophisticated, IoT big data management in a centralized architecture begins to suffer from single points of failure, poor scalability, high latency, and privacy issues. The blockchain can provide a continued integrity service for IoT big data management by identity, tamper-proof, fault-tolerant, confidentiality, etc. [73].

Another key BIoT application is related to communication domains. The blockchain is providing new insights and addressing some of the main challenges of many communication areas. For example, the blockchain can establish connections to various $5 \mathrm{G}$ networks and assist $5 \mathrm{G}$ networks to select the quickest access node for every user [74]. There is clear evidence that BIoT applications will play a major role in regard to IIoT in the future. As many researchers have observed, BIoT can revolutionize most of the current and future industrial applications in different sectors through the provision of a decentralized architecture [75]. In particular, the integration of blockchain with the IIoT will be a key enabler for many future technologies [76]. In the 
future, industrial BIoT applications will be an active field of research and hold important technical promise.

4.3. Limitations. Although the findings of this study are helpful for researchers to understand the current progress, hot spots, and future directions in the field of BIoT-related research, some limitations should be addressed. One limitation lies on the retrieval scope (SSCI and SCIE databases in WoS Core Collection), resulting in some important conference papers not being included. Other databases can be added in the future to verify the robustness of this study. Another limitation is that it included only articles written in English, which may lead to an underestimation regarding some important literature published in other languages.

\section{Conclusions}

In this article, we explore the recent progress in the field of BIoT-related research based on bibliometric analysis of publications in 2016-2020. Statistical results show a significant increase in the number of annual BIoT-related publications, peaking in 2020. China and the USA are the most productive and influential countries with the most publications, the highest citations, and the most cooperative countries. The keywords co-occurrence analysis indicates that hot issues around BIoT research can be categorized into four areas: security issues, core technologies, application dimensions, and transaction processes. According to the text mining analysis, the future research direction of BloT will focus more on computing paradigms and key applications. However, the results obtained can help researchers to delve deeper into the specific areas of this theme and offer the possibility of expanding collaboration for future researchers.

\section{Data Availability}

The data used to support the findings of the study are available within the article.

\section{Conflicts of Interest}

The authors declare that they have no conflicts of interest.

\section{Acknowledgments}

This work was supported in part by the National Social Science Fund of China under grant no. 16BJY087.

\section{References}

[1] D. E. Kouicem, A. Bouabdallah, and H. Lakhlef, "Internet of things security: a top-down survey," Computer Networks, vol. 141, no. 8, pp. 199-221, 2018.

[2] Cisco, "Cisco annual internet report," White paper, vol. 13, pp. 1-35, 2020.

[3] T. M. Fernandez-Carames and P. Fraga-Lamas, "A review on the use of blockchain for the internet of things," IEEE Access, vol. 6, pp. 32979-33001, 2018.

[4] A. Reyna, C. Martín, J. Chen, E. Soler, and M. Díaz, "On blockchain and its integration with IoT. Challenges and opportunities," Future Generation Computer Systems, vol. 88, no. 11, pp. 173-190, 2018.

[5] X. Wang, X. Zha, W. Ni et al., "Survey on blockchain for internet of things," Computer Communications, vol. 136, no. 2, pp. 10-29, 2019.

[6] Q. Wang, X. Zhu, Y. Ni, L. Gu, and H. Zhu, "Blockchain for the IoT and industrial IoT: a review," Internet of Things, vol. 10, no. 6, Article ID 100081, 2020.

[7] S. Nakamoto, Bitcoin: a peer-to-peer electronic cash system https://bitcoin.org/bitcoin.pdf, 2009.

[8] J. Yli-Huumo, D. Ko, S. Choi, S. Park, and K. Smolander, "Where is current research on blockchain technology? -A systematic review," PLoS One, vol. 11, no. 10, Article ID e0163477, 2016.

[9] F. Hawlitschek, B. Notheisen, and T. Teubner, "The limits of trust-free systems: a literature review on blockchain technology and trust in the sharing economy," Electronic Commerce Research and Applications, vol. 29, pp. 50-63, 2018.

[10] F. J. de Haro-Olmo, A. J. Varela-Vaca, and J. A. AlvarezBermejo, "Blockchain from the perspective of privacy and anonymisation: a systematic literature review," Sensors (Basel), vol. 20, no. 24, Article ID 7765152, 2020.

[11] P. Patil, M. Sangeetha, and V. Bhaskar, "Blockchain for IoT access control, security and privacy: a review," Wireless Personal Communications, vol. 117, no. 1, pp. 1-20, 2020.

[12] N. Kshetri, "Can blockchain strengthen the internet of things?” IT Professional, vol. 19, no. 4, pp. 68-72, 2017.

[13] K. Christidis and M. Devetsikiotis, "Blockchains and smart contracts for the internet of things," IEEE Access, vol. 4, pp. 2292-2303, 2016.

[14] M. C. K. Khalilov and A. Levi, "A survey on anonymity and privacy in bitcoin-like digital cash systems," IEEE Communications Surveys \& Tutorials, vol. 20, no. 3, pp. 2543-2585, 2018.

[15] M. Gutiérrez-Salcedo, M. Á. Martínez, J. A. Moral-Munoz, E. Herrera-Viedma, and M. J. Cobo, "Some bibliometric procedures for analyzing and evaluating research fields," Applied Intelligence, vol. 48, pp. 1275-1287, 2017.

[16] W. Iqbal, J. Qadir, G. Tyson, A. N. Mian, S.-u. Hassan, and J. Crowcroft, "A bibliometric analysis of publications in computer networking research," Scientometrics, vol. 119, no. 2, pp. 1121-1155, 2019.

[17] D. R. Raban and A. Gordon, "The evolution of data science and big data research: a bibliometric analysis," Scientometrics, vol. 122, no. 3, pp. 1563-1581, 2020.

[18] S. H. Zyoud et al., "Global research trends in microbiome-gutbrain axis during 2009-2018: a bibliometric and visualized study," BMC Gastroenterology, vol. 19, no. 1, Article ID 6716890, 2019.

[19] S. H. Zyoud, "Global scientific trends on aflatoxin research during 1998-2017: a bibliometric and visualized study," Journal of Occupational Medicine and Toxicology, vol. 14, Article ID 6873441, 2019.

[20] H. Guo, "Bibliometric analysis of internet of things based on CiteSpace,” IE\& EM, vol. 2019, pp. 276-283, 2020.

[21] D. Yu, W. Wang, W. Zhang, and S. Zhang, "A bibliometric analysis of research on multiple criteria decision making," Current Science, vol. 114, no. 4, pp. 747-758, 2018.

[22] Z.-C. Li, H.-J. Huang, and H. Yang, "Fifty years of the bottleneck model: a bibliometric review and future research directions," Transportation Research Part B: Methodological, vol. 139, pp. 311-342, 2020. 
[23] H. Liao, M. Tang, L. Luo, C. Li, F. Chiclana, and X.-J. Zeng, “A bibliometric analysis and visualization of medical big data research," Sustainability, vol. 10, no. 1, pp. 1-18, 2018.

[24] A. Firdaus, M. F. A. Razak, A. Feizollah, I. A. T. Hashem, M. Hazim, and N. B. Anuar, "The rise of "blockchain": bibliometric analysis of blockchain study," Scientometrics, vol. 120, no. 3, pp. 1289-1331, 2019.

[25] M. Dabbagh, M. Sookhak, and N. S. Safa, "The evolution of blockchain: a bibliometric study," IEEE Access, vol. 7, pp. 19212-19221, 2019.

[26] B. Muessigmann, H. von der Gracht, and E. Hartmann, "Blockchain technology in logistics and supply chain management-A bibliometric literature review from 2016 to january 2020," IEEE Transactions on Engineering Management, vol. 67, no. 4, pp. 988-1007, 2020.

[27] G. C. Nobre and E. Tavares, "Scientific literature analysis on big data and internet of things applications on circular economy: a bibliometric study," Scientometrics, vol. 111, no. 1, pp. 463-492, 2017.

[28] Y. Bouzembrak, M. Klüche, A. Gavai, and H. J. P. Marvin, "Internet of Things in food safety: literature review and a bibliometric analysis," Trends in Food Science \& Technology, vol. 94, pp. 54-64, 2019.

[29] A. Buchan, "Accountability and high impact journals in the health sciences," Publications, vol. 5, no. 1, pp. 1-12, 2017.

[30] N. M. Modak, "Forty years of computers \& chemical engineering: a bibliometric analysis," Computers \& Chemical Engineering, vol. 141, Article ID 106978, 2020.

[31] J. E. Hirsch, "An index to quantify an individual's scientific research output," Proceedings of the National Academy of Sciences, vol. 102, no. 46, pp. 16569-16572, 2005.

[32] C. Analytics, InCites Indicators Handbook https://clarivate. libguides.com/incites_ba, 2020.

[33] X. Zou, W. L. Yue, and H. L. Vu, "Visualization and analysis of mapping knowledge domain of road safety studies," Accident Analysis \& Prevention, vol. 118, pp. 131-145, 2018.

[34] R. K. Buter, E. C. M. Noyons, M. Van Mackelenbergh, and T. Laine, "Combining concept maps and bibliometric maps: first explorations," Scientometrics, vol. 66, no. 2, pp. 377-387, 2006.

[35] N. J. v. Eck and L. Waltman, "Software survey: VOSviewer, a computer program for bibliometric mapping," Scientometrics, vol. 84, no. 2, pp. 523-538, 2010.

[36] N. J. V. Eck and L. Waltman, Manual VOSviewer https:// www.vosviewer.com/documentation/Manual_VOSviewer_1. 6.15.pdf, 2020.

[37] N. M. A. M. Nadzar, A. Bakri, and R. Ibrahim, "A bibliometric mapping of Malaysian publication using Co-word analysis," International Journal of Soft Computing and Its Applications, vol. 9, no. 3, pp. 90-113, 2017.

[38] M. A. Khan and K. Salah, "IoT security: review, blockchain solutions, and open challenges," Future Generation Computer Systems, vol. 82, pp. 395-411, 2018.

[39] Z. Zheng, S. Xie, H. N. Dai, X. Chen, and H. Wang, "Blockchain challenges and opportunities: a survey," International Journal of Web and Grid Services, vol. 14, no. 4, pp. 352-375, 2018.

[40] M. M. Kessler, "Bibliographic coupling between scientific papers," American Documentation, vol. 14, no. 1, pp. 10-25, 1963.

[41] A. Perianes-Rodriguez, L. Waltman, and N. J. van Eck, "Constructing bibliometric networks: a comparison between full and fractional counting," Journal of Informetrics, vol. 10, no. 4, pp. 1178-1195, 2016.
[42] H. Small, "Co-citation in the scientific literature: a new measure of the relationship between two documents," Journal of the American Society for Information Science, vol. 24, no. 4, pp. 265-269, 1973.

[43] A. S. Patil, "Efficient privacy-preserving authentication protocol using PUFs with blockchain smart contracts," Computers \& Security, vol. 97, Article ID 101958, 2020.

[44] Q. S. He, Y. Xu, Z. B. Liu, J. H. He, Y. Sun, and R. Zhang, “A privacy-preserving Internet of Things device management scheme based on blockchain," International Journal of Distributed Sensor Networks, vol. 14, no. 11, Article ID 155014771880875, 2018.

[45] P. J. Taylor, T. Dargahi, A. Dehghantanha, R. M. Parizi, and K.-K. R. Choo, "A systematic literature review of blockchain cyber security," Digital Communications and Networks, vol. 6, no. 2, pp. 147-156, 2020.

[46] Y. Qian, Y. Jiang, J. Chen et al., "Towards decentralized IoT security enhancement: a blockchain approach," Computers \& Electrical Engineering, vol. 72, pp. 266-273, 2018.

[47] F. Li, D. Wang, Y. Wang et al., "Wireless communications and mobile computing blockchain-based trust management in distributed internet of things," Wireless Communications and Mobile Computing, vol. 2020, no. 5, 12 pages, Article ID 8864533, 2020.

[48] M. Li, F. R. Yu, P. Si, W. Wu, and Y. Zhang, "Resource optimization for delay-tolerant data in blockchain-enabled IoT with edge computing: a deep reinforcement learning approach," IEEE Internet of Things Journal, vol. 7, no. 10, pp. 9399-9412, 2020.

[49] C. Ye, W. Cao, and S. Chen, "Security challenges of blockchain in Internet of things: systematic literature review," Transactions on Emerging Telecommunications Technologies, vol. 11, Article ID ett.4177, 2020.

[50] S. Ahmed, M. A. Shah, and K. Wakil, "Blockchain as a trust builder in the smart city domain: a systematic literature review," IEEE Access, vol. 8, pp. 92977-92985, 2020.

[51] C. Pop, T. Cioara, M. Antal, I. Anghel, I. Salomie, and M. Bertoncini, "Blockchain based decentralized management of demand response programs in smart energy grids," Sensors (Basel), vol. 18, no. 1, Article ID 5796446, 2018.

[52] A. D. Dwivedi, G. Srivastava, S. Dhar, and R. Singh, "A decentralized privacy-preserving healthcare blockchain for IoT," Sensors (Basel), vol. 19, no. 2, Article ID 6359727, 2019.

[53] N. Kshetri, "1 Blockchain's roles in meeting key supply chain management objectives," International Journal of Information Management, vol. 39, pp. 80-89, 2018.

[54] B. Esmaeilian, "Blockchain for the future of sustainable supply chain management in Industry 4.0," Resources, Conservation and Recycling, vol. 163, no. 12, Article ID 105064, 2020.

[55] I. Mistry, S. Tanwar, S. Tyagi, and N. Kumar, "Blockchain for 5G-enabled IoT for industrial automation: a systematic review, solutions, and challenges," Mechanical Systems and Signal Processing, vol. 135, no. 1, Article ID 106382, 2020.

[56] U. Javaid, M. N. Aman, and B. Sikdar, "A scalable protocol for driving trust management in internet of vehicles with blockchain," IEEE Internet of Things Journal, vol. 7, no. 12, pp. 11815-11829, 2020.

[57] W. Nikolakis, L. John, and H. Krishnan, "How blockchain can shape sustainable global value chains: an evidence, verifiability, and enforceability (EVE) framework," Sustainability, vol. 10, no. 11, Article ID 103926, 2018.

[58] W. Lian, Q. Fan, B. Jia, Y. Liang, and J. P. Amezquita-Sanchez, "A blockchain prediction model on time, value, and purchase based on Markov chain and queuing theory in Stock trade," 
Mathematical Problems in Engineering, vol. 2020, Article ID 3984924, 13 pages, 2020.

[59] C. Alcaraz, J. E. Rubio, and J. Lopez, "Blockchain-assisted access for federated Smart Grid domains: coupling and features," Journal of Parallel and Distributed Computing, vol. 144, no. 10, pp. 124-135, 2020.

[60] Y. Yu, Y. Li, J. Tian, and J. Liu, "Blockchain-based solutions to security and privacy issues in the internet of things," IEEE Wireless Communications, vol. 25, no. 6, pp. 12-18, 2018.

[61] S. Wang, L. Ouyang, Y. Yuan, X. Ni, X. Han, and F.-Y. Wang, "Blockchain-Enabled smart contracts: architecture, applications, and future trends," IEEE Transactions on Systems, Man, and Cybernetics: Systems, vol. 49, no. 11, pp. 2266-2277, 2019.

[62] Hyperledger, Advancing Business Blockchain Adoption through Global Open Source Collaboration https://www. hyperledger.org/, 2020.

[63] BigchainDB, The Blockchain Database https://www. bigchaindb.com/, 2020.

[64] Stellar, Stellar Is an Open Network for Storing and Moving Money https://www.stellar.org/, 2020.

[65] Tendermint, Building the Most Powerful Tools for Distributed Networks https://tendermint.com/, 2020.

[66] N. J. Van Eck and L. Waltman, "Text mining and visualization using VOSviewer," ISSI Newsletter, vol. 7, no. 3, pp. 50-54, 2011.

[67] Z. Xu, W. Liu, J. Huang, C. Yang, J. Lu, and H. Tan, “Artificial intelligence for securing IoT services in edge computing: a survey," Security and Communication Networks, vol. 2020, no. 1, 13 pages, Article ID 8872586, 2020.

[68] X. Huang, D. Ye, R. Yu, and L. Shu, "Securing parked vehicle assisted fog computing with blockchain and optimal smart contract design," IEEE/CAA Journal of Automatica Sinica, vol. 7, no. 2, pp. 426-441, 2020.

[69] P. Kumar, "A Distributed framework for detecting DDoS attacks in smart contract-based Blockchain-IoT Systems by leveraging Fog computing," Transactions on Emerging Telecommunications Technologies, vol. 9, pp. 1-31, 2020.

[70] Y. Liu, J. Zhang, and J. Zhan, "Privacy protection for fog computing and the internet of things data based on blockchain," Cluster Computing, vol. 10, 2020.

[71] S. Singh, "Convergence of blockchain and artificial intelligence in IoT network for the sustainable smart city," Sustainable Cities and Society, vol. 63, no. 12, Article ID 102364, 2020.

[72] R. Gupta, A. Kumari, and S. Tanwar, "Fusion of blockchain and artificial intelligence for secure drone networking underlying 5G communications," Transactions on Emerging Telecommunications Technologies, vol. 32, no. 1, Article ID e4176, 2020.

[73] Y. E. Oktian, S.-G. Lee, and B.-G. Lee, "Blockchain-based continued integrity service for IoT big data management: a comprehensive design," Electronics, vol. 9, no. 9, Article ID 9091434, 2020.

[74] G. Praveen, V. Chamola, V. Hassija, and N. Kumar, "Blockchain for 5G: a prelude to future telecommunication," IEEE Network, vol. 34, no. 6, pp. 106-113, 2020.

[75] K.-K. R. Choo, Z. Yan, and W. Meng, "Editorial: blockchain in industrial IoT applications: security and privacy advances, challenges, and opportunities," IEEE Transactions on Industrial Informatics, vol. 16, no. 6, pp. 4119-4121, 2020.

[76] B. Seok, J. Park, and J. H. Park, "A lightweight hash-based blockchain architecture for industrial IoT," Applied Sciences, vol. 9, no. 18, Article ID 9183740, 2019. 